\title{
Prognostic Implication of Preoperative Behavior Changes in Patients with Primary High-Grade Meningiomas
}

\author{
Andrej Vranic ${ }^{1}$ and Frederic Gilbert ${ }^{2}$ \\ ${ }^{1}$ Department of Neurosurgery, University Medical Centre Ljubljana (UMC Ljubljana), Zaloska 7, 1000 Ljubljana, Slovenia \\ ${ }^{2}$ Ethics \& Bionics/Nanomedicine, Australian Centre of Excellence in Electromaterials Science, University of Tasmania, \\ 7000 Hobart, Australia
}

Correspondence should be addressed to Andrej Vranic; andrejvranic@yahoo.fr

Received 19 August 2013; Accepted 20 November 2013; Published 21 January 2014

Academic Editors: V. Pistoia, B. Schaller, and C. J. Vecht

\begin{abstract}
Copyright (C) 2014 A. Vranic and F. Gilbert. This is an open access article distributed under the Creative Commons Attribution License, which permits unrestricted use, distribution, and reproduction in any medium, provided the original work is properly cited.
\end{abstract}

\begin{abstract}
High-grade meningiomas are rare extra-axial tumors, frequently causing brain invasion and prominent brain edema. Patients harboring high-grade meningiomas occasionally present with behavior changes. Data about frequency and prognostic importance of preoperative behavior changes in patients with high-grade meningiomas is missing. 86 patients with primary high-grade meningiomas were analyzed. Statistical analysis was performed to determine correlation of preoperative behavior changes with tumor location, preoperative brain edema, tumor cleavability, tumor grade, Ki67 proliferation index, and microscopic brain invasion. Survival analysis was performed. 30 (34.9\%) patients presented with preoperative behavior changes. These changes were more frequent with male patients $(P=0.066)$ and patients older than 55 years $(P=0.018)$. They correlated with frontal location $(P=0.013)$, tumor size $(P=0.023)$, microscopic brain invasion $(P=0.015)$, and brain edema $(P=0.006)$. Preoperative behavior changes did not correlate with duration of symptoms, tumor cleavability, tumor malignancy grade, and Ki67 proliferation index. They were not significantly related to overall survival or recurrence-free survival of patients with primary high-grade meningiomas. Preoperative behavior changes are frequent in patients harboring primary high-grade meningiomas. They correlate with tumor size, microscopic brain invasion, and brain edema. Preoperative behavior changes do not predict prognosis in patients with primary high-grade meningiomas.
\end{abstract}

\section{Introduction}

Meningiomas represent the most frequently diagnosed primary central nervous system tumors in adults over 35 years, accounting for $33.8 \%$ in the USA between 2004 and 2006 [1]. Women are affected twice as commonly as men. According to the WHO classification of brain tumors [2], about $3 \%$ of meningiomas are malignant (WHO Grade III) and about 20\% are atypical (WHO Grade II). The rest account for benign meningiomas (WHO Grade I).

While benign meningiomas in general are encapsulated and slowly growing, high-grade (Grade II/III) meningiomas often recur. Recurrence rates up to $80 \%$ for Grade II and up to $100 \%$ for Grade III meningiomas in 20 years have been reported [3]. Tumor grade, microscopical brain invasion, high mitotic count, and parasagittal location are negative prognostic factors of recurrence-free survival of patients with high-grade meningiomas $[3,4]$.

Most frequent presenting signs of high-grade meningioma patients are epileptic seizures, hemiparesis, and headache [5]. Mean duration of symptoms is 7.5 months prior to diagnosis [6]. There is no difference in duration of symptoms between primary and recurrent high-grade meningiomas [7]. So far, no type of presenting symptoms has been confirmed as a prognostic sign for meningioma recurrence. Both lowand high-grade meningioma patients may also present with behavior changes, frequently described as psychoorganic syndrome. Data about frequency and prognostic importance of preoperative behavior changes in patients with brain tumors, including patients with high-grade meningiomas, is missing [8]. 
In the present study, we analyzed 86 consecutive patients with primary high-grade meningiomas, treated at a single institution. Our aim was to assess the frequency, correlation with other factors, and prognostic importance of preoperative behavior changes in patients with primary high-grade meningiomas, which, to the best of our knowledge, have so far not been evaluated yet.

\section{Materials and Methods}

2.1. Patients. 86 consecutive patients with primary highgrade meningiomas diagnosed between 1990 and 2005 were included in the study. In the same time period, 790 primary benign meningiomas were diagnosed. All patients were surgically treated at the Department of Neurosurgery, University Medical Centre, Ljubljana, Slovenia. Selection of patients was based on original pathological reports. All original histological slides were reviewed when the original diagnosis was either Grade II or Grade III meningioma. Cases were reviewed without knowledge of clinical outcome and other clinical data. Microscopical brain invasion was assessed separately, and Ki67 proliferative index, which is a measure of cellular proliferation, was determined using routine immunohistochemical protocols.

Clinical data were retrieved from the patients' medical records. The following clinical data were collected: tumor site (L/R), location and size, preoperative signs and symptoms, duration of symptoms, presence of brain edema on preoperative MR scans, tumor cleavability, and extent of surgical resection. Brain edema was graded as absent, mild, or severe. Data on cleavability were obtained from original operative reports with special emphasis on the tumor/brain interface. Meningiomas were classified as noncleavable when pial invasion was observed by the surgeon and cleavable when dissection in the extrapial plane could be performed [9].

Data on preoperative behavior changes were also retrieved from patients' medical records. Patients were classified as having preoperative behavior changes if any data about behavior changes was noted in their history.

Follow-up data were collected for all patients, including recurrence-free survival (RFS; time from surgical resection to local recurrence) and overall survival (OS; time from surgical resection to death). The dates of patients' death were collected from the Cancer Registry of Slovenia. No attempts were made to determine the cause of death.

2.2. Statistical Analysis. Statistical analysis was performed with SPSS 16.0 for Windows (SPSS Inc., Chicago, IL). The differences between groups in relation to other clinicopathological parameters were analyzed by the Mann-Whitney and the Kruskal-Wallis tests. Survival analysis was performed by the Cox model and survival curves were estimated using the Kaplan-Meier method. The final multivariate model was achieved using a stepwise backward elimination of nonsignificant variables.

\section{Results}

86 consecutive patients with primary high-grade meningiomas were analyzed. Among 86 high-grade meningiomas cases, 76 meningiomas (88\%) were Grade II and 10 (12\%) were Grade III. 42 patients were male (49\%) and 44 (51\%) were female.

Out of 86 patients analyzed, clinical data was available for 83 patients. Behavior of 30 patients (34.9\%) was described by their relatives as being changed. Out of these 30 patients, behavior changes were a presenting symptom in 12 cases (14\%). Other patients presented with headaches (23 patients; $26.7 \%$ ), hemiparesis (22 patients; $25.6 \%$ ), epileptic fits (20 patients; $23.3 \%$ ), and cranial nerves palsy (6 patients; $7 \%$ ). In the group with behavior changes, patients were mostly suffering from aggressiveness, although other changes like apathy or hypersexuality were also described. Clinical and pathological characteristics of 86 high-grade meningiomas are shown in Table 1.

Patients with behavior changes were older (mean 57.1 years) than patients with normal preoperative behavior (mean 53.7 years) $(P=0.017)$. Behavior changes tended to be more frequent in male patients: they were present in 19 out of $42(47.5 \%)$ male and 11 out of $44(27.5 \%)$ female patients $(P=0.066)$. They were found more frequently in larger tumors $(P=0.023)$ and were strongly associated with presence of brain edema $(P=0.006)$. Preoperative behavior changes were present in $31(53.4 \%)$ cases with mild edema and in 17 (29.3\%) cases with severe brain edema, while absent in all cases with no brain edema. They were also strongly correlated to tumor location, being more frequent in patients with tumors located frontally or frontobasally $(P=0.013)$. Preoperative behavior changes were less frequent in tumors located parasagittally $(P=0.008)$ and were not correlated to the site $(\mathrm{L} / \mathrm{R})$ of the tumor $(P=0.298)$. While there was a strong correlation of behavior changes with microscopic brain invasion $(P=0.015)$, no correlation with tumor cleavability as assessed by the surgeon was noticed. There was also no correlation between preoperative behavior changes and duration of symptoms, WHO Grade of the tumor, or Ki67 proliferation index. Correlation of several factors with preoperative behavior changes is shown in Table 2.

Patients were followed until death or for a median of 96 months (range from 28 to 204 months). The 5- and 10year recurrence-free survival (RFS) for the entire group of 86 high-grade meningiomas was $60 \%(95 \% \mathrm{CI}, 48-71)$ and $55 \%$ (95\% CI, 43-68), respectively. The 5- and 10-year overall survival (OS) for the entire group was 76\% (95\% CI, 67$86)$ and $56 \%$ (95\% CI, 44-68), respectively. The univariate Cox analyses of the RFS and OS are shown in Table 3. On multivariate analysis, brain invasion and parasagittal-falcine location were independent negative predictors of RFS, while male gender and parasagittal-falcine location were the only parameters associated with decreased OS. There were no significant differences in RFS or OS between patients with or without preoperative behavior changes.

\section{Discussion}

Psychoorganic syndrome is a vague definition of behavior changes caused by a morphologically proven lesion in the brain. It has been described in patients with brain tumors, including meningiomas [10-13], revealing a dilemma 
TABLE 1: Clinical characteristics of 86 cases of high-grade meningiomas.

\begin{tabular}{|c|c|}
\hline Parameter & $n^{*}(\%)$ \\
\hline Gender & 86 \\
\hline Male & $42(48.8)$ \\
\hline Female & $44(51.2)$ \\
\hline Age & 86 \\
\hline Range, $13.5-85.4$ years & - \\
\hline Mean, 55.0 years & - \\
\hline Median, 57.2 years & - \\
\hline Tumor size & 78 \\
\hline Range, $2.0-8.5 \mathrm{~cm}$ & - \\
\hline Mean, $4.9 \mathrm{~cm}$ & - \\
\hline Median, $5.0 \mathrm{~cm}$ & - \\
\hline Tumor location & 86 \\
\hline Parasagittal-falcine & $26(30.2)$ \\
\hline Convexity & $35(40.7)$ \\
\hline Base & $24(27.9)$ \\
\hline Frontal/frontobasal & $16(18.6)$ \\
\hline Tumor site & 86 \\
\hline Left & 37 \\
\hline Right & 31 \\
\hline Bilateral & 18 \\
\hline Duration of signs/symptoms & 80 \\
\hline Range, $0-36$ months & - \\
\hline Mean, 7.5 months & - \\
\hline Median, 4.0 months & - \\
\hline Presenting signs/symptoms & 83 \\
\hline Headache & $23(25.9)$ \\
\hline Hemiparesis & $22(36.6)$ \\
\hline Seizures & $20(24.7)$ \\
\hline Behavior changes & $12(14)$ \\
\hline Cranial nerve palsy & $6(11.1)$ \\
\hline Behavior changes & 83 \\
\hline Present & $30(36.1)$ \\
\hline Absent & $53(73.9)$ \\
\hline Edema & 58 \\
\hline Absent & $10(17.2)$ \\
\hline Mild & $31(53.4)$ \\
\hline Severe & $17(29.3)$ \\
\hline Cleavability & 42 \\
\hline Cleavable & $18(42.9)$ \\
\hline Noncleavable & $24(57.1)$ \\
\hline
\end{tabular}

${ }^{*}$ The information on some parameters was not available for all patients.

whether neuroradiologic imaging should be a part of the routine diagnostic procedure in all psychiatric patients.

Most frequent behavior changes in patients with brain tumors are slowness, apathy, aggressiveness, hypersexuality, social inappropriateness, poor judgment, and lack of planning and rarely hallucinations and other psychotic symptoms [11]. Preoperative behavior changes are frequent findings in
TABLE 2: Correlation of several factors with preoperative behavior changes.

\begin{tabular}{ll}
\hline Brain edema & 0.006 \\
Nonparasagittal location & 0.008 \\
Frontal/frontobasal location & 0.013 \\
Brain invasion & 0.015 \\
Age & 0.018 \\
Tumor size & 0.023 \\
Sex & 0.066 \\
Cleavability & 0.233 \\
Tumor site L/R & 0.298 \\
Ki67 & 0.404 \\
Duration of symptoms & 0.626 \\
WHO Grade & 0.650 \\
\hline
\end{tabular}

patients with butterfly gliomas (i.e., glioblastoma multiforme affecting both frontal lobes) or benign olfactory meningiomas $[10,11]$. These two conditions affect both frontal lobes, causing malfunction of the prefrontal cortex. Several cases of unilateral frontal or frontobasal tumors causing behavior changes have been described [13-15]. Correlation with frontal or frontobasal location was also confirmed in our study, even if the tumor was unilateral. However, preoperative behavior changes are not limited to patients with frontal lobe tumors. Rare cases of unusual acquired aggressive and antisocial behavior correlated with frontal, temporal, thalamic, hypothalamic, or left parietal tumors have been reported in the literature [16-22]. So far, reviews have demonstrated no correlation between specific psychiatric symptoms and tumor location or histological type [23]. Since it was impossible to specify the behavior changes, no correlation between the type of behavior changes and the location of the tumor could be performed in our retrospective study.

We have shown that preoperative behavior changes, although not prognostically important, present a frequent finding in patients with high-grade meningiomas. According to our results, preoperative behavior changes mostly correlate with tumor invasion in the surrounding brain, as well as with brain edema. In high-grade meningioma patients, brain edema is more the result of tumor invasion than venous compression. Behavior changes do not correlate with other tumor malignancy properties, such as cellular proliferation, measured with the Ki67 proliferation index, neither do they correlate with the meningioma grade. Therefore, behavior changes may be the result of tumor cell invasion into the brain parenchyma as well as of brain edema forming in the invaded brain. Less probably, they may develop as the result of direct pressure on the brain parenchyma by a rapidly proliferating tumor.

Behavior changes may be the only initial manifestation of meningiomas [8]. Our study confirmed that in $14 \%$ they are a presenting symptom in patients with high-grade meningiomas. Since growth rate of high-grade meningiomas is relatively slow compared to malignant gliomas, the onset of behavior changes is slow, behavior changes are subtle, 
TABLE 3: Univariate survival analysis of 86 high-grade meningiomas.

\begin{tabular}{|c|c|c|c|c|c|c|c|}
\hline \multirow{2}{*}{ Variable } & \multirow{2}{*}{$n$} & \multicolumn{3}{|c|}{ Recurrence-free survival } & \multicolumn{3}{|c|}{ Overall survival } \\
\hline & & HR & $95 \% \mathrm{CI}$ & $P$ value & HR & $95 \% \mathrm{CI}$ & $P$ value \\
\hline Sex (male versus female) & 86 & 1.9 & $0.9-4.0$ & 0.077 & 1.9 & $1.0-3.8$ & 0.064 \\
\hline Age $(\geq 55$ versus $<55)$ & 86 & 0.9 & $0.5-1.8$ & 0.776 & 3.19 & $1.5-6.7$ & 0.002 \\
\hline Location (parasagittal-falcine versus others) & 86 & 2.3 & $1.1-4.7$ & 0.021 & 1.5 & $0.8-3.0$ & 0.238 \\
\hline WHO Grade (III versus II) & 86 & 2.0 & $0.8-5.3$ & 0.145 & 1.7 & $0.6-4.3$ & 0.293 \\
\hline Brain invasion (present versus absent) & 37 & 7.2 & $0.9-55.7$ & 0.059 & 4.4 & $1.0-19.2$ & 0.049 \\
\hline Ki67 (>4\% versus $\leq 4 \%)$ & 86 & 2.7 & $1.1-6.7$ & 0.027 & 1.9 & $0.9-4.0$ & 0.104 \\
\hline Behavior changes (present versus absent) & 83 & 0.7 & $0.3-1.7$ & 0.514 & 1.0 & $0.5-2.0$ & 0.112 \\
\hline
\end{tabular}

and patients with high-grade meningiomas may first be referred to psychiatrists [24]. In these cases, if radiological imaging is not performed, the accurate diagnosis may only emerge when the tumor has grown large enough to compress the brainstem and disturb other brain functions, such as consciousness [25].

A recent study reported significant risk for brain tumors in the first 1-2 years after admittance to a psychiatric hospital [26]. Nevertheless, studies have reported no raised incidence of intracranial tumors among patients manifesting schizophrenia, affective illness, or neurotic symptoms [27]. A very small incidence of positive radiological findings in psychiatric patients, except for brain atrophy, does not give good reason for routine CT and MRI brain scans in all hospitalized psychiatric patients [28].

In our study, we have showed that patients with highgrade meningiomas frequently manifest behavior changes, sometimes even as presenting symptoms. Since prognosis in these patients is worse than prognosis of patients with benign meningiomas-time to recurrence in malignant meningiomas is less than one year and no chemotherapy is effective-it is important not to delay the diagnosis. Early diagnosis gives the best chance of successful treatment to high-grade meningioma patients. The possibility of a patient suffering from behavior changes due to high-grade meningioma, initially being admitted to a psychiatric facility, illustrates how initial evaluation is critical. In these rare cases, transition from psychiatric to neurosurgical care management should be accelerated and surgical treatment should be performed immediately. In the process of diagnostics of patients with subtle behavior changes, the idea of high-grade meningioma must not be neglected.

Methodological shortcoming of this study is especially the retrospective nature of the study, without the use of a structured questionnaire to determine the nature of behavior changes. Correlation between the type of behavior changes and tumor location would add a lot to understanding the nature of psychiatric symptoms in patients with brain tumors.

\section{Conclusions}

Preoperative behavior changes are frequent in patients harboring primary high-grade meningiomas. They correlate with tumor size, microscopic brain invasion, and brain edema. Preoperative behavior changes do not predict prognosis in patients with primary high-grade meningiomas.

\section{Conflict of Interests}

The authors declare that there is no conflict of interests regarding the publication of this paper.

\section{Acknowledgment}

This research was partially funded by the Australian Centre of Excellence for Electromaterials Science (ACES) CE0561616.

\section{References}

[1] C. Kruchko, Central Brain Tumor Registry of the United States, vol. 32, CBTRUS, Hinsdale, Ill, USA, 2010.

[2] A. Perry, D. N. Louis, B. W. Scheithauer et al., “. Meningiomas," in World Health Organisation Classification of Tumours: WHO Classification of Tumours of the Central Nervous System, D. N. Louis, H. Ohgaki, and O. D. Wiestler, Eds., pp. 164-172, IARC Press, Lyon, France, 2007.

[3] J. Jaaskelainen, M. Haltia, and E. Laasonen, "The growth rate of intracranial meningiomas and its relation to histology. An analysis of 43 patients," Surgical Neurology, vol. 24, no. 2, pp. 165-172, 1985.

[4] A. Durand, F. Labrousse, A. Jouvet et al., "WHO grade II and III meningiomas: a study of prognostic factors," Journal of NeuroOncology, vol. 95, no. 3, pp. 367-375, 2009.

[5] A. Mahmood, D. V. Caccamo, F. J. Tomecek, G. M. Malik, P. M. Black, and J. J. Kepes, "Atypical and malignant meningiomas: a clinicopathological review," Neurosurgery, vol. 33, no. 6, pp. 955-963, 1993.

[6] A. Vranic, M. Popovic, A. Cör, B. Prestor, and J. Pizem, "Mitotic count, brain invasion, and location are independent predictors of recurrence-free survival in primary atypical and malignant meningiomas: a study of 86 patients," Neurosurgery, vol. 67, no. 4, pp. 1124-1132, 2010.

[7] J. Ayerbe, D. R. Lobato, J. De la Cruz et al., "Risk factors predicting recurrence in patients operated on for intracranial meningioma. A multivariate analysis," Acta Neurochirurgica, vol. 141, no. 9, pp. 921-932, 1999.

[8] R. K. Gupta and R. Kumar, "Benign brain tumours and psychiatric morbidity: a 5-years retrospective data analysis," 
Australian and New Zealand Journal of Psychiatry, vol. 38, no. 5, pp. 316-319, 2004.

[9] J. E. Alvernia and M. P. Sindou, "Preoperative neuroimaging findings as a predictor of the surgical plane of cleavage: prospective study of 100 consecutive cases of intracranial meningioma," Journal of Neurosurgery, vol. 100, no. 3, pp. 422-430, 2004.

[10] F. Gilbert, A. Vranic, and S. Hurst, "Involuntary \& voluntary invasive brain surgery: ethical issues related to acquired aggressiveness," Neuroethics, vol. 6, no. 1, pp. 115-128, 2013.

[11] J. Barrash, D. Tranel, and S. W. Anderson, "Acquired personality disturbances associated with bilateral damage to the ventromedial prefrontal region," Developmental Neuropsychology, vol. 18, no. 3, pp. 355-381, 2000.

[12] D. K. L. Dae Kyu Lee, D. G. K. Dong Gyu Kim, G. Choe, J. G. Chi, and H.-W. Jung, "Chordoid meningioma with polyclonal gammopathy. Case report," Journal of Neurosurgery, vol. 94, no. 1, pp. 122-126, 2001.

[13] T. L. Avery, "Seven cases of frontal tumour with psychiatric presentation," British Journal of Psychiatry, vol. 119, no. 548, pp. 19-23, 1971.

[14] R. Hunter, W. Blackwood, and J. Bull, "Three cases of frontal meningiomas presenting psychiatrically," British Medical Journal, vol. 3, article 9, 1968.

[15] J. M. Burns and R. H. Swerdlow, "Right orbitofrontal tumor with pedophilia symptom and constructional apraxia sign," Archives of Neurology, vol. 60, pp. 437-440, 2003.

[16] B. L. Miller, J. L. Cummings, and H. McIntyre, "Hypersexuality or altered sexual preference following brain injury," Journal of Neurology Neurosurgery and Psychiatry, vol. 49, no. 8, pp. 867873, 1986.

[17] R. Caplan, Y. Comair, D. A. Shewmon, L. Jackson, H. T. Chugani, and W. J. Peacock, "Intractable seizures, compulsions, and coprolalia: a pediatric case study," Journal of Neuropsychiatry and Clinical Neurosciences, vol. 4, no. 3, pp. 315-319, 1992.

[18] L. J. Villano, N. Mlinarevich, K. S. Watson, H. H. Engelhard, and L. Anderson-Shaw, "Aggression in a patient with primary brain tumor: ethical implications for best management," Journal of Neuro-Oncology, vol. 94, no. 2, pp. 293-296, 2009.

[19] R. M. Haugh and W. R. Markesbery, "Hypothalamic astrocytoma. Syndrome of hyperphagia, obesity, and disturbances of behavior and endocrine and autonomic function," Archives of Neurology, vol. 40, no. 9, pp. 560-563, 1983.

[20] P. Nakaji, H. S. Meltzer, S. A. Singel, and J. F. Alksne, "Improvement of aggressive and antisocial behavior after resection of temporal lobe tumors," Pediatrics, vol. 112, no. 5, p. e430, 2003.

[21] P. W. Kaplan, D. A. Kerr, and A. Olivi, "Ictus expectoratus: a sign of complex partial seizures usually of non-dominant temporal lobe origin," Seizure, vol. 8, no. 8, pp. 480-484, 1999.

[22] G. D. Partlow, R. Carpio-O’Donovan, D. Melanson, and T. M. Peters, "Bilateral thalamic glioma: review of eight cases with personality change and mental deterioration," American Journal of Neuroradiology, vol. 13, no. 4, pp. 1225-1230, 1992.

[23] S. Madhusoodanan, D. Danan, and D. Moise, "Psychiatric manifestations of brain tumors: diagnostic implications," Expert Review of Neurotherapeutics, vol. 7, no. 4, pp. 343-349, 2007.

[24] C. Dumas-Duport, Tumeurs cerebrales chez les malades mentaux [Ph.D. thesis], Faculté de Medicine de Paris, Paris, France, 1970.

[25] R. S. Maurice-Williams and G. Dunwoody, "Late diagnosis of frontal meningiomas presenting with psychiatric symptoms," British Medical Journal, vol. 296, no. 6639, pp. 1785-1786, 1988.
[26] M. E. Benros, T. M. Laursen, S. O. Dalton, and P. B. Mortensen, "Psychiatric disorder as a first manifestation of cancer: a 10-year population-based study," International Journal of Cancer, vol. 124, no. 12, pp. 2917-2922, 2009.

[27] M. A. Ron, "Psychiatric manifestations of frontal lobe tumours," British Journal of Psychiatry, vol. 155, pp. 735-738, 1989.

[28] K. Hrabric, V. Jukic, P. Bilic et al., "Neuroradiologic diagnostics in patients hospitalized in Vrapce Psychiatric Hospital," Lijec Vjesn, vol. 131, pp. 122-125, 2009. 


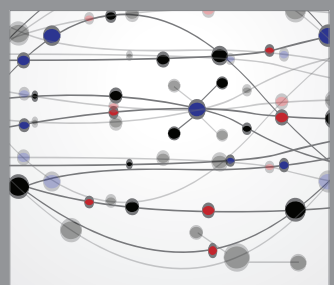

The Scientific World Journal
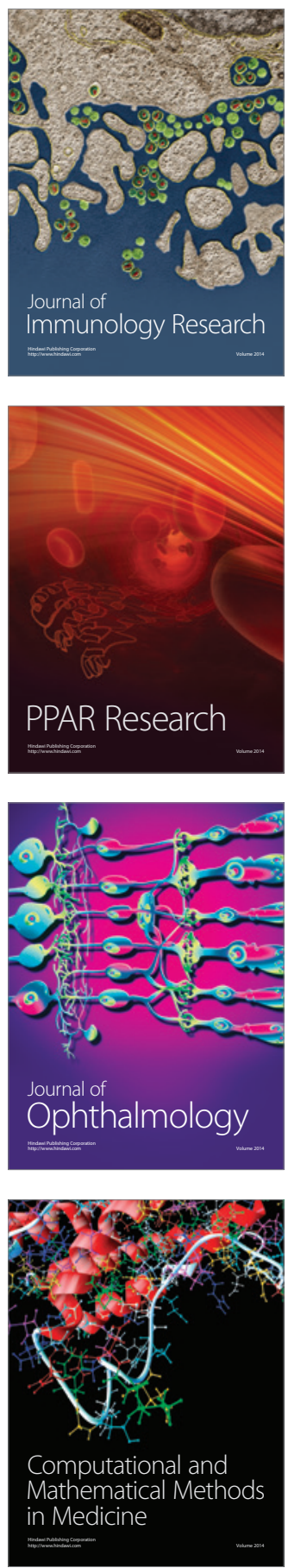

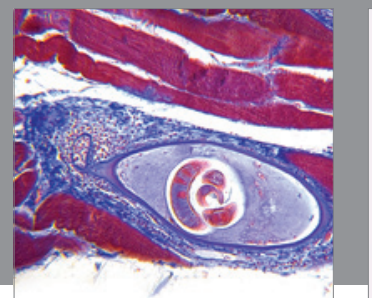

Gastroenterology

Research and Practice
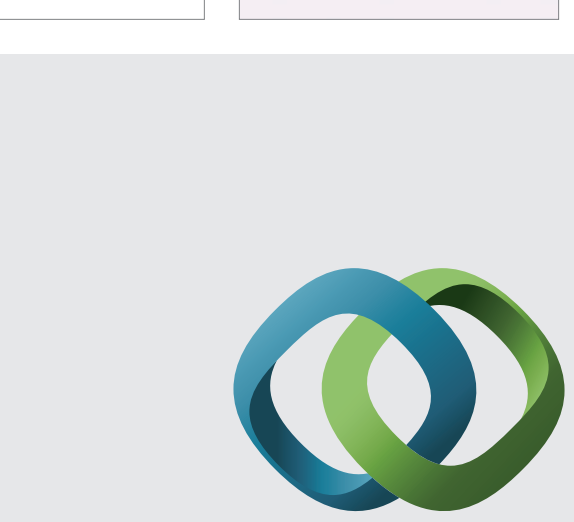

\section{Hindawi}

Submit your manuscripts at

http://www.hindawi.com
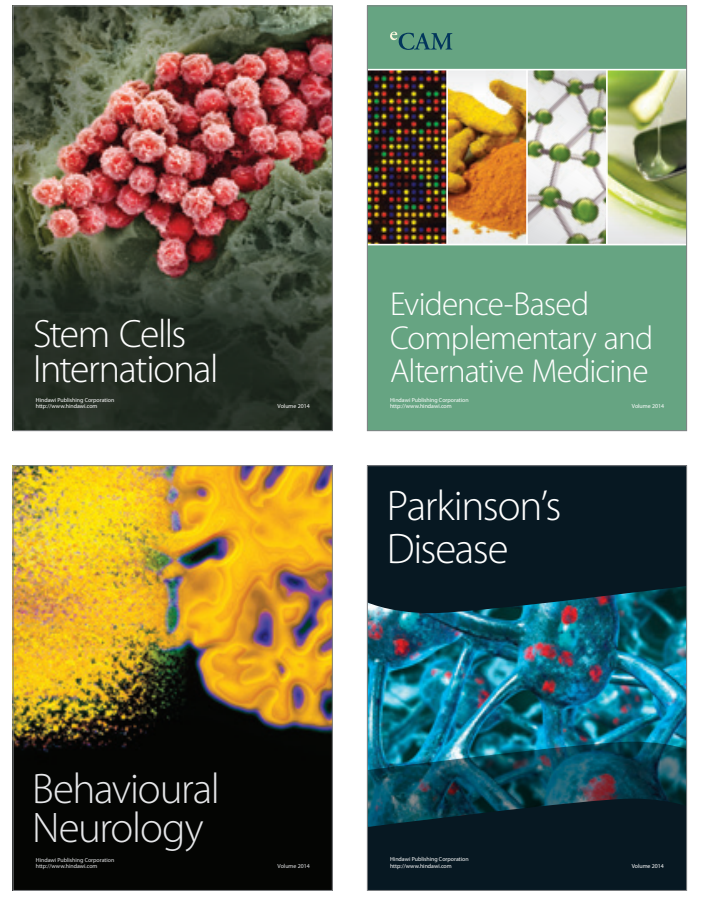
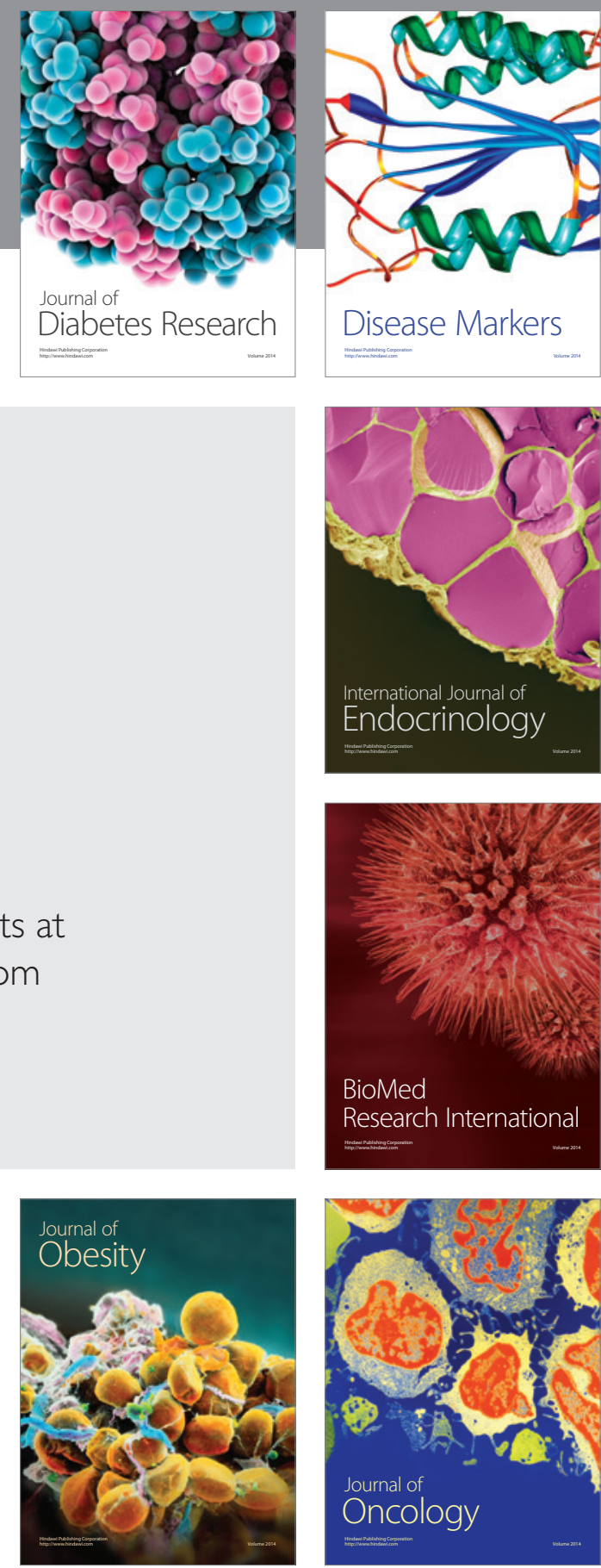

Disease Markers
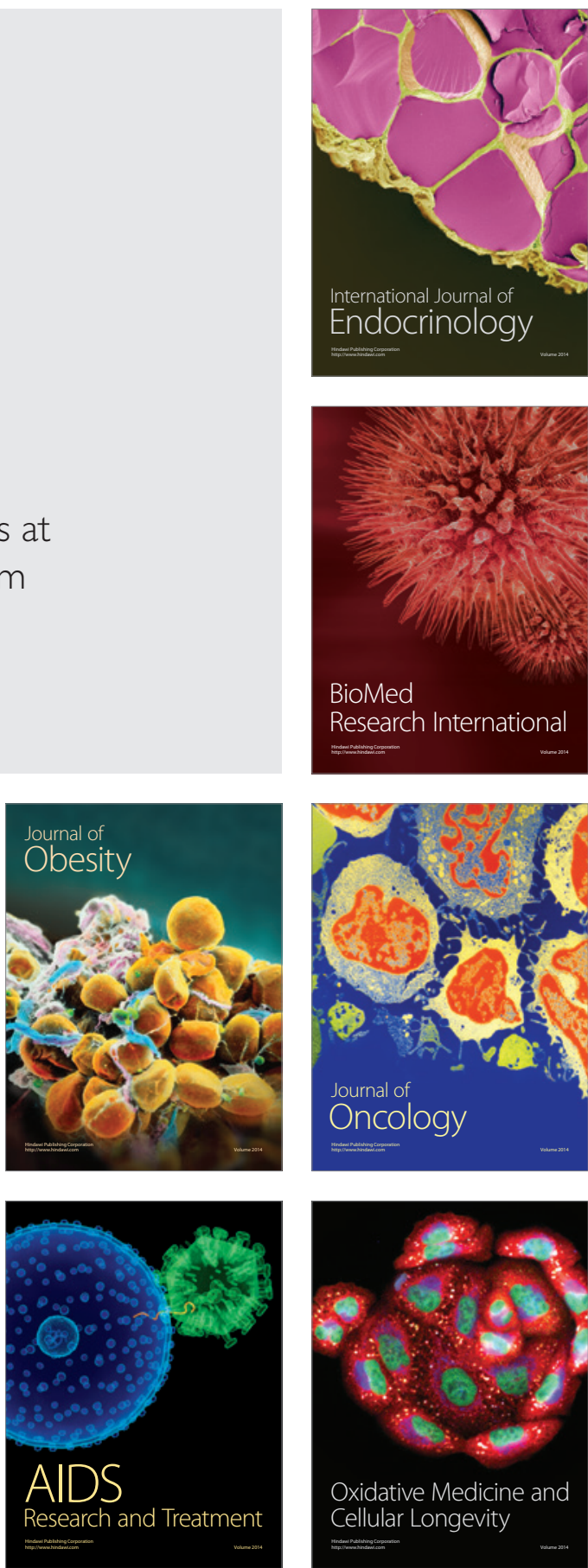